\title{
ON ABSOLUTE BOREL-TYPE METHODS OF SUMMABILITY
}

\section{BORWEIN}

1. Introduction. Suppose throughout that $l, a_{n}(n=0,1, \cdots)$ are arbitrary complex numbers, that $\lambda>0$ and $\mu$ is real, and that $N$ is a nonnegative integer such that $\lambda N+\mu \geqq 1$. Let $s_{-1}=0, s_{n}=\sum_{v=0}^{n} a_{\nu}$;

$$
a_{\lambda, \mu}(x)=\sum_{n=N}^{\infty} \frac{a_{n} x^{\lambda n+\mu-1}}{\Gamma(\lambda n+\mu)}, \quad s_{\lambda, \mu}(x)=\sum_{n=N}^{\infty} \frac{s_{n} x^{\lambda n+\mu-1}}{\Gamma(\lambda n+\mu)} .
$$

Borel-type methods of summability are defined as follows: The series $\sum_{0}^{\infty} a_{n}$ is said to be

(i) summable $(B, \lambda, \mu)$ to $l$, if $s_{\lambda, \mu}(x)$ is finite for all $x \geqq 0$ and $\lambda e^{-x} s_{\lambda, \mu}(x) \rightarrow l$ as $x \rightarrow \infty$;

(i) summable $\left(B^{\prime}, \lambda, \mu\right)$ to $l$, if $a_{\lambda, \mu}(x)$ is finite for all $x \geqq 0$ and $\int_{0}^{v} e^{-x} a_{\lambda, \mu}(x) d x+s_{N-1} \rightarrow l$ as $y \rightarrow \infty$;

(ii) absolutely summable $(B, \lambda, \mu)$, or summable $|B, \lambda, \mu|$, to $l$, if the series is summable $(B, \lambda, \mu)$ to $l$ and $e^{-x} s_{\lambda, \mu}(x)$ is of bounded variation on $[0, \infty)$;

(ii)' absolutely summable $\left(B^{\prime}, \lambda, \mu\right)$, or summable $\left|B^{\prime}, \lambda, \mu\right|$, to $l$, if the series is summable $(B, \lambda, \mu)$ to $l$ and $\int_{0}^{y} e^{-x} a_{\lambda, \mu}(x) d x$ is of bounded variation on $[0, \infty)$.

Note that the methods $(B, 1,1)$ and $\left(B^{\prime}, 1,1\right)$ are respectively equivalent to the standard Borel exponential and integral methods $B$ and $B^{\prime}$.

The object of this paper is to establish the following absolute summability analogue of a known inclusion theorem for ordinary Borel-type summability ([2, Result I] and [1, Theorem 2]; see also [4]):

Theorem. If $\alpha>\lambda$, the series $\sum_{0}^{\infty} a_{n}$ is summable $\left|B^{\prime}, \alpha, \beta\right|$ to $l$, and $a_{\lambda, \mu}(x)$ is finite for all $x \geqq 0$, then the series is summable $\left|B^{\prime}, \lambda, \mu\right|$ to $l$.

It is known that [1, Lemma 4] $a_{\lambda, \mu}(x)$ is finite for all $x \geqq 0$ if and only if $s_{\lambda, \mu}(x)$ is finite for all $x \geqq 0$; and that [3, Theorem 17] a series is summable $\left|B^{\prime}, \lambda, \mu\right|$ to $l$ if and only if it is summable $|B, \lambda, \mu+1|$ to $l$. Hence " $B$ " may be replaced by " $B$ " in the theorem.

\section{Preliminary results.}

Lemma 1. If $\delta>0$ and a series is summable $\left|B^{\prime}, \alpha, \beta\right|$ to $l$ then it is

Received by the editors January 20, 1969. 
summable $\left|B^{\prime}, \alpha, \beta+\delta\right|$ to $l$.

This lemma is known [5].

Lemma 2. If $\alpha>\lambda$ and $\beta / \alpha \geqq \mu / \lambda$, then there is a function $\psi$, continuous on $(0, \infty)$, such that

$$
\begin{gathered}
\frac{\Gamma(\alpha n+\beta)}{\Gamma(\lambda n+\mu)}=\int_{0}^{\infty} t^{n} \psi(t) d t \quad(n \geqq N), \\
\int_{0}^{\infty} t^{n}|\psi(t)| d t=O\left(\frac{\Gamma(\alpha n+\beta)}{\Gamma(\lambda n+\mu)}\right) \quad(n \geqq N),
\end{gathered}
$$

and, for any $\delta>0$,

$$
u^{\rho(\alpha-\lambda)} \psi\left(u^{\alpha-\lambda}\right)=O\left(e^{-k u}\left(u^{1 / 2}+u^{-\sigma-\delta}\right)\right) \quad(0<u<\infty)
$$

where $\rho=1-(\beta-\mu) /(\alpha-\lambda), \sigma=\beta-\alpha \mu / \lambda, k=((\alpha-\lambda) / \lambda)(\lambda / \alpha)^{\alpha /(\alpha-\lambda)}$.

Proof. Let $h(s)=\Gamma(\alpha s+\beta) / \Gamma(\lambda s+\mu)$. Then by Stirling's theorem (see [2, p. 129]), there is a positive constant $C$ such that

$$
h(s)=e^{(\alpha \log \alpha-\lambda \log \lambda-\alpha+\lambda) s} s^{(\alpha-\lambda) s+\beta-\mu}\{C+O(1 /|s|)\}
$$

when $|s|$ is large and $\operatorname{Re} s>-\mu / \lambda$. Since $N>-\mu / \lambda$, it follows from the proof of Lemma 4 in [2], with $\sigma_{0}=-\mu / \lambda, \nu=N$, that there is a function $\phi$, continuous on $(0, \infty)$, such that

$$
\begin{gathered}
h(n)=\int_{0}^{\infty} t^{n-N} \phi(t) d t \quad(n \geqq N) ; \\
\int_{0}^{\infty} t^{n-N}|\phi(t)| d t=O(h(n)) \quad(n \geqq N) ; \\
t^{-N} \phi(t)=O\left(t^{\mu / \lambda-1-\delta /(\alpha-\lambda)}\right)=O\left(t^{-(\sigma+\delta) /(\alpha-\lambda)}\right) \quad \text { as } \quad t \rightarrow 0+;
\end{gathered}
$$

and

$$
t^{-N} \phi(t) \sim K e^{-k t^{1 /(\alpha-\lambda)}} t^{-\rho+1 / 2(\alpha-\lambda)} \quad \text { as } \quad t \rightarrow \infty,
$$

where $K$ is a positive constant.

Putting $\psi(t)=t^{-N} \phi(t)$, we obtain the conclusions of Lemma 2 .

3. Proof of the theorem. Let

$$
\begin{gathered}
\gamma=\alpha / \lambda, \quad \rho=1-(\beta-\mu) /(\alpha-\lambda), \quad \sigma=\beta-\gamma \mu, \\
k=(\gamma-1) \gamma^{\gamma /(1-\gamma)}, \quad \delta=(\gamma-1)^{2} / \gamma .
\end{gathered}
$$

By Lemma 1, we may suppose, without loss in generality that $\beta \geqq \gamma \mu$, i.e. that $\sigma \geqq 0$. 
The main hypotheses of the theorem are that

$$
\int_{0}^{\infty} e^{-y}\left|a_{\alpha, \beta}(y)\right| d y<\infty,
$$

and that

$$
a_{\lambda, \mu}(x) \text { is finite for all } x \geqq 0 .
$$

Let $\psi$ be the function specified in Lemma 2. Then, for $0<x<\infty$,

$$
\begin{aligned}
a_{\lambda, \mu}(x) & =\sum_{n=N}^{\infty} \frac{a_{n} x^{\lambda n+\mu-1}}{\Gamma(\alpha n+\beta)} \frac{\Gamma(\alpha n+\beta)}{\Gamma(\lambda n+\mu)}=\sum_{n=N}^{\infty} \frac{a_{n} x^{\lambda n+\mu-1}}{\Gamma(\alpha n+\beta)} \int_{0}^{\infty} t^{n} \psi(t) d t \\
& =x^{\mu-1+(1-\beta) / \gamma} \int_{0}^{\infty} t^{(1-\beta) / \alpha} \psi(t) d t \sum_{n=N}^{\infty} \frac{a_{n}\left(x^{1 / \gamma} t^{1 / \alpha}\right)^{\alpha n+\beta-1}}{\Gamma(\alpha n+\beta)} \\
& =x^{\mu-1+(1-\beta) / \gamma} \int_{0}^{\infty} t^{(1-\beta) / \alpha} \psi(t) a_{\alpha, \beta}\left(x^{1 / \gamma} t^{1 / \alpha}\right) d t,
\end{aligned}
$$

the inversion of sum and integral being legitimate since, by (2), there is a constant $M$ such that

$$
\sum_{n=N}^{\infty} \frac{\left|a_{n}\right| x^{\lambda n+\mu-1}}{\Gamma(\alpha n+\beta)} \int_{0}^{\infty} t^{n}|\psi(t)| d t<M \sum_{n=N}^{\infty} \frac{\left|a_{n}\right| x^{\lambda n+\mu-1}}{\Gamma(\lambda n+\mu)},
$$

which is finite by (5).

Substitute $t=x^{-\lambda} y^{\alpha}, d t=\alpha x^{-\lambda} y^{\alpha-1} d y$ in the final integral in (6) to get

$$
a_{\lambda, \mu}(x)=\alpha x^{\mu-\lambda-1} \int_{0}^{\infty} y^{\alpha-\beta} a_{\alpha, \beta}(y) \psi\left(x^{-\lambda} y^{\alpha}\right) d y \quad(0<x<\infty),
$$

and hence

$$
\int_{0}^{\infty} e^{-x}\left|a_{\lambda, \mu}(x)\right| d x
$$

$$
\leqq \alpha \int_{0}^{\infty}\left|a_{\alpha, \beta}(y)\right| y^{\alpha-\beta} d y \int_{0}^{\infty} e^{-x} x^{\mu-\lambda-1}\left|\psi\left(x^{-\lambda} y^{\alpha}\right)\right| d x .
$$

Now substitute $x=y v^{\gamma-1}, d x=(\gamma-1) y v^{\gamma-2} d v$ in the inner integral on the right-hand side of (7) to get

$$
\begin{aligned}
& \int_{0}^{\infty} e^{-x}\left|a_{\lambda, \mu}(x)\right| d x \\
& \leqq \alpha(\gamma-1) \int_{0}^{\infty}\left|a_{\alpha, \beta}(y)\right| d y \int_{0}^{\infty} e^{-y v^{\gamma-1} \nu^{-\sigma-1}(y / v)^{\rho(\alpha-\lambda)}\left|\psi\left((y / v)^{\alpha-\lambda}\right)\right| d v .}
\end{aligned}
$$


Consequently, by (3), there is a constant $M_{1}$ such that

$$
\int_{0}^{\infty} e^{-x}\left|a_{\lambda, \mu}(x)\right| d x \leqq M_{1} \int_{0}^{\infty} e^{-y}\left|a_{\alpha, \beta}(y)\right| I(y) d y
$$

where

$$
I(y)=\int_{0}^{\infty} e^{-\left(v^{\gamma}-v+k\right) y / v}\left\{(y / v)^{1 / 2}+(y / v)^{-\sigma-\delta}\right\} v^{-\sigma-1} d v .
$$

Let $f(v)=v^{\gamma}-v+k, c=\gamma^{1 /(1-\gamma)}$. Then $f(c)=f^{\prime}(c)=0, f(v)>0$ when $v>0, v \neq c$, and

$$
f(v) /(v-c)^{2} \rightarrow f^{\prime \prime}(c) / 2=\gamma(\gamma-1) c^{\gamma-2} / 2 \text { as } \quad v \rightarrow c .
$$

Hence there are positive constants $p, q, r$ such that

$$
f(v) \geqq p, \quad v^{-\gamma} f(v) \geqq q \quad \text { when } 0<v<c / 2 \quad \text { or } \quad v>3 c / 2 ;
$$

and $f(v) \geqq r v(v-c)^{2}$ when $c / 2<v<3 c / 2$.

It follows that, for $y>0$,

$$
\begin{aligned}
& I(y) \leqq y^{1 / 2} \int_{c / 2}^{3 c / 2} e^{-r(v-c)^{2}} v^{-\sigma-3 / 2} d v+y^{1 / 2} \int_{0}^{\infty} e^{-p y / v} v^{-\sigma-3 / 2} d v \\
& +y^{-\sigma-\delta} \int_{c / 2}^{3 c / 2} v^{\delta-1} d v+y^{-\sigma-\delta} \int_{0}^{\infty} e^{-q y v^{\gamma-1}} v^{\delta-1} d v \\
& \leqq 2\left(\frac{c}{2}\right)^{-\sigma-3 / 2} y^{1 / 2} \int_{0}^{c / 2} e^{-r t^{2}} d t+y^{-\sigma} \int_{0}^{\infty} e^{-p t t^{\sigma-1 / 2}} d t \\
& +y^{-\sigma-\delta}\left(\frac{3 c}{2}\right)^{\delta} \delta^{-1}+y^{-\sigma-\delta \gamma /(\gamma-1)} \int_{0}^{\infty} e^{-q t t^{-1+\delta /(\gamma-1)}} d t \\
& \leqq M_{2}\left(1+y^{-\sigma}+y^{-\sigma-\delta}+y^{-\sigma-\gamma+1}\right)
\end{aligned}
$$

where $M_{2}$ is a constant; i.e.

$$
I(y)=O(1) \quad(1 \leqq y<\infty),
$$

and, since $\delta=(\gamma-1)^{2} / \gamma<\gamma-1$,

$$
I(y)=O\left(y^{-\sigma-\gamma+1}\right) \quad(0<y<1) .
$$

In virtue of $(10)$, we have

$$
\begin{aligned}
I(y) a_{\alpha, \beta}(y) & =I(y) \sum_{n=N}^{\infty} \frac{a_{n} y^{\alpha n+\beta-1}}{\Gamma(\alpha n+\beta)} \\
& =O\left(y^{-\sigma-\gamma+1+\alpha N+\beta-1}\right)=O\left(y^{\gamma(\lambda N+\mu-1)}\right), \\
& =O(1) \quad(0<y<1) .
\end{aligned}
$$


It follows from (4), (9) and (11) that

$$
\int_{0}^{\infty} e^{-y}\left|a_{\alpha, \beta}(y)\right| I(y) d y<\infty .
$$

Consequently, by (8),

$$
\int_{0}^{\infty} e^{-x}\left|a_{\lambda, \mu}(x)\right| d x<\infty,
$$

i.e. $\sum_{0}^{\infty} a_{n}$ is summable $|B, \lambda, \mu|$.

Further, by the inclusion theorem for ordinary Borel-type summability referred to in $\S 1$, the $|B, \lambda, \mu|$ sum of the series $\sum_{0}^{\infty} a_{n}$ is the same as its $|B, \alpha, \beta|$ sum. This completes the proof.

\section{REFERENCES}

1. D. Borwein, Relations between Borel-type methods of summability, J. London Math. Soc. 35 (1960), 65-70. MR 22 \#6958.

2. - On methods of summability based on integral functions. II, Proc. Cambridge Philos. Soc. 56 (1960), 125-131. MR 22 \#6957.

3. D. Borwein and B. L. R. Shawyer, On Borel-type methods, Tôhoku Math. J. (2) 18 (1966), 283-298. MR 35 \#3312.

4. I. J. Good, Some relations between certain methods of summation of infinite series, Proc. Cambridge Philos. Soc. 38 (1942), 144-165. MR 3, 297.

5. B. L. R. Shawyer, On the relation between Abel and Borel-type methods of summability, Proc. Amer. Math. Soc. 22 (1969), 15-19.

UNIVERSITY OF WESTERN ONTARIO 